\title{
The IFRD1 (57460C>T Polymorphism) Gene: A Negative Report in Cystic Fibrosis Clinical Severity
}

\author{
Fernando Augusto de Lima Marson ${ }^{1,2 *}$, Aline Roberta Bariani Marcelino , Luciana Montes Rezende ${ }^{1}$, Antônio Fernando Ribeiro², José \\ Dirceu Ribeiro² and Carmen Sílvia Bertuzzo ${ }^{1}$
}

${ }^{1}$ Department of Medical Genetics, University of Campinas, Unicamp, School of Medical Sciences, FCM, Tessália Vieira de Camargo, 126 , Cidade Universitária "Zeferino Vaz", Campinas, SP, Brazil

${ }^{2}$ Department of Pediatrics, University of Campinas, Unicamp, School of Medical Sciences, FCM, Tessália Vieira de Camargo, 126, Cidade Universitária "Zeferino Vaz", Campinas, SP, Brazil

\begin{abstract}
Cystic fibrosis (CF) is an autosomal recessive disease caused by more than 1,900 mutations in the Cystic Fibrosis Transmembrane Conductance Regulator (CFTR) gene. In CF, one intriguing aspect is that patients, with same CFTR mutation, can have high clinical variability. Thus, the CFTR genotype does not seem to be the only determining factor in the clinical severity modulation. Therefore, the modifier genes and the environment must be considered. The IFRD1 (Interferon-related developmental regulator 1) gene, acts on the immune system and in the recruitment of immune cells, and consequently could be a modulator. In our data we included 88 CF patients, diagnosed by CFTR mutation screening and positive sweat test. The $57460 \mathrm{C}>\mathrm{T}$ polymorphism screening in the IFRD1 gene was made by polymerase chain reaction associated to enzymatic digestion. A genotypic comparison was performed with $23 \mathrm{CF}$ clinical variables. The data was analyzed by the SPSS program considering $\alpha=0.05$. The patients were analyzed considering the CFTR genotype characteristic by mutation class. In our data $64.77 \%$ of patients had mutations of classes I, II or III in the CFTR gene. The IFRD1 polymorphism frequency was 28 (12.99\%), $35(75.32 \%)$ and $25(11.69 \%)$ to the CC, CT and TT genotypes, respectively. In our study, the 57460C>T polymorphism in the IFRD1 gene was not associated with the CF clinical variables. The analysis was performed with and without consideration of the CFTR genotype, and after correction for multiple testing (Bonferroni test), no positive association was observed in both cases. Taking into account our results, in the CF patients population analyzed, there were no associations of the $57460 \mathrm{C}>$ T polymorphism in the IFRD1 gene with the CF clinical variables.
\end{abstract}

Keywords: Cystic fibrosis; IFRD1 gene; Genotype; Phenotype; Variability; Lung disease; Polymorphism; CFTR gene

\section{Introduction}

The cystic fibrosis (CF) is a monogenic, autosomal and recessive disease, with wide clinical variability [1-3]. Children with same CFTR (Cystic Fibrosis Transmembrane Regulator) genotype, siblings or twins, show wide clinical variability [4], however, monozygotic twins have a higher clinical concordance than dizygotic twins. In this case, the modifier genes should be considered [5-7] principally genes involved in the control of infection, immunity and inflammation. The expression of modifier genes, conditioned by their polymorphisms, can act: (i) in ion transport by without CFTR channel, on a molecular level, (ii) by altering chlorine conduction, (iii) in controlling the splicing and expression of the CFTR gene, (iv) by altering the mucociliary clearance, and (v) in the repair of epithelial tissue [2,6-8].

Our group has studied CF severity in association with modifier genes, including: $M B L-2, T G F-\beta 1, C D 14$ [9], GSTM1, GSTT1 [10], $A C E$ [11], ADRB2 [12], TCF7L2 [13], COX-2 [14] and ADRA2A [15]. In our studies, the polymorphisms are associated with clinical variables including clinical markers of the pulmonary and digestive disease.

The IFRD1 (Interferon-Related Developmental Regulator 1) gene, region $7 \mathrm{q} 31.1$, has 13 exons, with $52 \mathrm{~Kb}$, transcribed with 1,834 bases pair, and is responsible for encoding a protein with 451 amino acids [16]. The correct function of IFRD1 protein is dependent of the histone deacetylase that is expressed in the late of the neutrophils differentiation, being important in neutrophil function [17,18]. The single sequence polymorphism, rs7817 [exchanging a cytosine to thymine at position 57460], in the 3'UTR region of the IFRD1 gene, had the heterozygous genotype $(\mathrm{CT})$ associated with worse lung function than the homozygous (CC and TT). Although the IFRD1 gene is located on chromosome 7, as is the CFTR gene, both genes have independent segregation [17].

In CF patients, the neutrophils are recruited continuously in the airways, causing persistent inflammatory response [19]. As the severity of the inflammatory response varies, even among patients with identical CFTR genotype, there is a need to study genes involved in the neutrophil production and maturation in CF [20]. A few studies related the IFRD1 gene as CF modifier gene, considering its ability to modulate the amplitude of the immune response of neutrophils [18-20].

In this study, we selected the IFRD1 $(57460 \mathrm{C}>\mathrm{T})$ polymorphism with expression related to the immune system. The IFRD1 protein is expressed in mature neutrophils and is able to interact with the histone deacetylase enzyme [18], acting in cellular differentiation and oxidative stress. Since CF pulmonary disease is characterized by neutrophilic inflammation and oxidative stress, the IFRD1 action can exert a key role in regulating airway inflammation [17]. In this context, the aim of

*Corresponding author: Fernando Augusto de Lima Marson, Department of Medical Genetics, University of Campinas, Unicamp, School of Medical Sciences FCM, Tessália Vieira de Camargo, 126, Cidade Universitária "Zeferino Vaz", Campinas, SP, Brazil, Tel: ++55 019 35218902; Fax: ++55 019 35218909; E-mail: fernandolimamarson@hotmail.com

Received May 23, 2013; Accepted June 14, 2013; Published June 17, 2013

Citation: de Lima Marson FA, Bariani Marcelino AR, Rezende LM, Ribeiro AF, Ribeiro JD, et al. (2013) The IFRD1 (57460C>T Polymorphism) Gene: A Negative Report in Cystic Fibrosis Clinical Severity. J Mol Genet Med 7: 058 doi:10.4172/1747-0862.1000058

Copyright: (C) 2013 de Lima Marson FA, et al. This is an open-access article distributed under the terms of the Creative Commons Attribution License, which permits unrestricted use, distribution, and reproduction in any medium, provided the original author and source are credited 
this study was to analyze the polymorphism $57460 \mathrm{C}>\mathrm{T}$ in the IFRD1 gene in association with 27 clinical variables in CF patients.

\section{Method}

\section{Patient and methods}

This was a cross-sectional study conducted in a university center for CF care between 2011 and 2012. All CF patients were invited participants of the study. CF patients without data or informed consent were not included. The CF diagnosis was confirmed by two doses of sodium and chloride from the sweat with values greater than $60 \mathrm{mEq} / \mathrm{L}$. In a patient's cohort, the CFTR mutation was identified. No patient had diagnosis made by neonatal screening test.

Eighty eight patients were selected for the study. Patients' DNA was obtained by phenol-chloroform extraction. The DNA concentration used for analysis was $50 \mathrm{ng} / \mathrm{mL}$, evaluated using GE NanoVue ${ }^{\mathrm{TM}}$ Spectrophotometer (GE Healthcare Biosciences, Pittsburgh, USA).

\section{Clinical variables}

The clinical variables were employed: (i) clinical scores (Shwachman-Kulczycki, Kanga and Bhalla) [21]; (ii) body mass index (BMI) for the patients older than 19 years of age the BMI= weight/ (height $)^{2}$ formula was used; for the remaining patients: WHO ANTHRO program (children 0 - under 5 years old) and WHO ANTHRO PLUS program (children 5 - under 19 years old) were used (http://www. who.int/en/); (iii) patient age; (iv) time for the diagnosis (according to sodium and chloride dosage); (v) first clinical symptoms (digestive and pulmonary); (vi) time for the 1st colonization by Pseudomonas aeruginosa; (vii) bacteria in the respiratory airways ( $P$. aeruginosa mucoid and no mucoid, Achromobacter xylosoxidans, Burkolderia cepacia and Staphylococcus aureus); (viii) transcutaneous hemoglobin oxygen saturation; (ix) spirometry; (x) comorbidities.

Spirometry was performed in patients older than 7 years old, using the CPFS/D spirometer (MedGraphics, Saint Paul, Minnesota, USA). Data was recorded by the PF BREEZE software version 3.8B for Windows 95/98/NT [22] and the following variables were included: forced vital capacity [FVC (\%)], forced expiratory volume in the first second $\left[\mathrm{FEV}_{1}(\%)\right]$ ratio between $\mathrm{FEV}_{1}$ and $\mathrm{FVC}(\%)\left[\mathrm{FEV}_{1} / \mathrm{FVC}(\%)\right]$ and forced expiratory flow between 25 and $75 \%$ of the $\mathrm{FVC}\left[\mathrm{FEF}_{25-75} \%\right]$.

The comorbidities included in the study were nasal polyps (diagnosed by physical examination and/or rhinoscopy), osteoporosis (diagnosed by bone densitometry), meconium ileus (diagnosed by meconium presence in the birth), diabetes mellitus type 2 (diagnosed by glucose tolerance exam) and pancreatic insufficiency (diagnosed by steatocrit).

This study was approved by the Institutional Ethics Committee from University of Campinas - Faculty of Medical Sciences (\#052/2011), and all patients signed a consent form before beginning the study.

\section{The CFTR mutation identification}

The CFTR mutation identification was performed by polymerase chain reaction (PCR) (F508del) and fragment length polymorphism method (G542X, R1162X, R553X, G551D and N1303K). Some mutations in CF patients were obtained by sequencing or MLPA (Multiplex Ligation-dependent Probe Amplification) analysis: 1717$\mathrm{G}>\mathrm{A}$ and I618T. For sequencing and MLPA, we used MegaBace1000 sequencer (GE Healthcare Biosciences, Pittsburgh, USA).

The CFTR genotype was used as a correction factor for statistical analysis. All mutations identified were included in the class I, II or III of the CFTR gene. Other identified mutations, class IV (P205S) were not included in statistical analysis.

\section{Analysis of $57460 \mathrm{C}>\mathrm{T}$ polymorphism in the IFRD1 gene}

The PCR reaction for amplification of the $547 \mathrm{bp}$ fragment of the IFRD1 gene was performed with bidistilled water, 10x Taq buffer with $\left(\mathrm{NH}_{4}\right)_{2} \mathrm{SO}_{4}, \mathrm{MgCl}_{2}(25 \mathrm{mM})$, dNTP (25 mM each nitrogenous base), primers ( 0.2 pmol - sense primer: 5'-AGATAAGAGAGCAGATGTT-3' and antisense primer: 5'-GCTGTCTTCATAAATAAAT-3'), Taq polymerase $(5 \mathrm{U})$ and genomic DNA $(50 \mathrm{ng} / \mathrm{mL})$. The annealing temperature was $62^{\circ} \mathrm{C}$.

After PCR, enzymatic digestion was made with the BstNI enzyme (New England BioLabs) at $60^{\circ} \mathrm{C}$ for 14 hours following the manufacturer's recommendations. The reaction was analyzed on polyacrylamide gel $(12 \%)$ with a voltage of $180 \mathrm{~V}$ for 4 hours. The gel was stained in ethidium bromide solution and visualized on the Typhoon $^{\text {Tx }}$ scanner (GE Healthcare, Pittsburgh, USA). According to fragments observed the genotype was identified, as follows: TT $(444+$ $113 \mathrm{bp})$, TC $(444+326+118+103 \mathrm{bp})$ and CC $(326+118+103 \mathrm{bp})$.

\section{Statistical analysis}

Statistical analysis was performed by Statistical Package for Social Sciences (SPSS) software v.21.0 (IBM Corp. Released 2012. IBM SPSS Statistics for Windows, Version 21.0. Armonk, NY: IBM Corp.), OpenEpi [23] and R version 2.12 (Comprehensive R Archive Network, 2011). The statistical power calculation for the sample was performed by GPower 3.1 software [24] demonstrating statistical power above $80 \%$ for the analysis.

The data were compared using the $\chi^{2}$ and Fisher exact test for categorical variables and the Mann-Whitney and Kruskal-Wallis tests for numerical variables.

We adopted the values of $<$ alpha $>0.05$ for all statistical analysis.

The data distribution that showed high standard deviation was analyzed by median value. The variables that were adjusted by median to short (more severe) and longtime were: patient's age ( $\leq 154$ and $>$ 154 months), time for the diagnosis $\leq 24$ and $>24$ months), onset of the pulmonary ( $\leq 6$ and $>6$ months) and digestive symptoms ( $\leq 3$ and $>3$ months), and time for the first isolated $P$. aeruginosa ( $\leq 3$ and $>3$ months).

In order to avoid spurious data due to the multiple tests [25], the significance level $\alpha$ was adjusted by Bonferroni correction ( $\alpha$ $0.05 /$ number of tests).

\section{Results}

The description of the population examined in the study is shown in the table 1 for all clinical variables included in the study.

The genotypic frequency of CFTR mutations and polymorphisms are described in the table 2 . The analyzed polymorphism is in HardyWeinberg equilibrium.

The table 3 shows the p-values, corrected and uncorrected, reported for all analyzes performed, considering all patients included in the study, and patients with two CFTR mutations identified.

The tables 4-6 shows the categorical variables and in the table 7, the numerical variables, regardless of the CFTR mutations and considering the distribution for the CFTR gene according to the presence of 
Citation: de Lima Marson FA, Bariani Marcelino AR, Rezende LM, Ribeiro AF, Ribeiro JD, et al. (2013) The IFRD1 (57460C>T Polymorphism) Gene: A Negative Report in Cystic Fibrosis Clinical Severity. J Mol Genet Med 7: 058. doi:10.4172/1747-0862.1000058

Page 3 of 8

\begin{tabular}{|c|c|}
\hline Sex - masculine & $48 \%(86)^{\#}$ \\
\hline Caucasian & $91.5 \%(161)^{\#}$ \\
\hline Age & $166.98 \pm 11.766$ months $(87-468 \text { months })^{*}$ \\
\hline BMI - thinness and accentuated thinness & $13.6 \%(12)^{\#}$ \\
\hline One Class I, II or III identified mutation & $34.09 \%(30)^{\#}$ \\
\hline Two Class I, II or III identified mutation & $64.77 \%(57)^{\#}$ \\
\hline First clinical manifestation & $5.58 \pm 1.222$ months $(0-39 \text { months })^{*}$ \\
\hline Age at diagnosis & $36.86 \pm 9.368$ months $(1-379 \text { months })^{*}$ \\
\hline Onset of digestive symptoms & $21.74 \pm 9.300$ months $(0-381 \text { months })^{*}$ \\
\hline Onset of pulmonary symptoms & $13.63 \pm 4.543$ months $(0-187 \text { months })^{*}$ \\
\hline $\mathrm{SpO}_{2}$ & $95.07 \pm 0.812(66-99)^{*}$ \\
\hline Bhalla score & $8.53 \pm 0.742(0-23)^{*}$ \\
\hline Kanga score & $18.86 \pm 0.851(11-40)^{*}$ \\
\hline Shwachman-Kulczycki score & $66.98 \pm 2.227(20-90)^{*}$ \\
\hline FVC(\%) & $82.49 \pm 3.194(29-135)^{*}$ \\
\hline $\mathrm{FEV}_{1}(\%)$ & $74.67 \pm 3.424(19-132)^{*}$ \\
\hline $\mathrm{FEV}_{1} / \mathrm{FVC}$ & $84.26 \pm 2.034(37-100)^{*}$ \\
\hline $\mathrm{FEF}_{25-75} \%$ & $61.77 \pm 4.461(8-121)^{\star}$ \\
\hline Nasal Polyps & $12.50 \%(11)^{\#}$ \\
\hline Diabetes mellitus & $18.20 \%(16)^{\#}$ \\
\hline Osteoporosis & $14.80 \%(13)^{\#}$ \\
\hline Pancreatic insufficiency & $96.90 \% \%(85)^{\#}$ \\
\hline Meconium ileus & $17.00 \%(15)^{\#}$ \\
\hline First isolated $P$. aeruginosa & $53.12 \pm 10.557$ months ( $4-379$ months) \\
\hline$P$. aeruginosa status ${ }^{1}$ & $63.60 \%(56)^{\#}$ \\
\hline$P$. aeruginosa mucoid status ${ }^{1}$ & $46.60 \%(41)^{\#}$ \\
\hline B. cepacia status ${ }^{1}$ & $19.30 \%(17)^{\#}$ \\
\hline A. $x$ losoxidans status ${ }^{1}$ & $14.80 \%(13)^{\#}$ \\
\hline S. aureus status ${ }^{1}$ & $81.20 \%(72)^{\#}$ \\
\hline
\end{tabular}

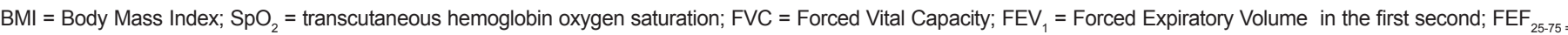
Forced Expiratory Flow between 25 and $75 \%$ of FVC. 1. Based on 3 Consecutive positive respiratory cultures.

\#Percentage (Number of patients)

${ }^{*}$ Continuous variables expressed as mean $\pm \mathrm{SD}$ (range)

Table 1: Clinical features of Cystic Fibrosis patients included in the study.

\begin{tabular}{|c|c|c|c|c|c|c|c|c|c|}
\hline \multirow{2}{*}{ Gene } & \multirow{2}{*}{$\begin{array}{l}\text { Chromosome } \\
\text { position }\end{array}$} & \multirow{2}{*}{ Location } & \multirow{2}{*}{ Variation } & \multicolumn{3}{|c|}{ Genotype } & \multirow{2}{*}{ MAF } & \multirow{2}{*}{$x^{2}$} & \multirow{2}{*}{$\mathbf{p}^{*}$} \\
\hline & & & & $\mathrm{C} / \mathrm{C}$ & $\mathrm{C} / \mathrm{T}$ & $T / T$ & & & \\
\hline IFRD $1,57460 \mathrm{C}>\mathrm{T}$ & $7 q 31.1$ & $\begin{array}{l}3 \text { untranslated } \\
\text { region }\end{array}$ & $\mathrm{C}>\mathrm{T}$ & $28(12.99 \%)$ & $35(75.32 \%)$ & $25(11.69 \%)$ & 0.48 & 3.65 & $>0.05^{1}$ \\
\hline \multicolumn{2}{|l|}{ CFTR mutation } & \multicolumn{2}{|c|}{$\mathbf{N}$} & \multicolumn{6}{|c|}{ Frequency } \\
\hline \multicolumn{2}{|l|}{ F508del/F508del } & \multicolumn{2}{|c|}{36} & \multicolumn{6}{|c|}{$40.90 \%$} \\
\hline \multicolumn{2}{|l|}{ F508del/G542X } & \multicolumn{2}{|c|}{10} & \multicolumn{6}{|c|}{$11.40 \%$} \\
\hline \multicolumn{2}{|l|}{ F508del/R1162X } & \multicolumn{2}{|c|}{2} & \multicolumn{6}{|c|}{$2.28 \%$} \\
\hline \multicolumn{2}{|l|}{ F508del/N1303K } & \multicolumn{2}{|c|}{3} & \multicolumn{6}{|c|}{$3.42 \%$} \\
\hline \multicolumn{2}{|l|}{ F508del/R553X } & \multicolumn{2}{|c|}{1} & \multicolumn{6}{|c|}{$1.14 \%$} \\
\hline \multicolumn{2}{|l|}{ F508del/1717-1G>A } & \multicolumn{2}{|c|}{1} & \multicolumn{6}{|c|}{$1.14 \%$} \\
\hline \multicolumn{2}{|l|}{ G542X/R1162X } & \multicolumn{2}{|c|}{1} & \multicolumn{6}{|c|}{$1.14 \%$} \\
\hline \multicolumn{2}{|l|}{ F508del/2184insA } & \multicolumn{2}{|c|}{1} & \multicolumn{6}{|c|}{$1.14 \%$} \\
\hline F508del/duplication & n $6 \mathrm{~B}$ to 16 & & & & & $1.14 \%$ & & & \\
\hline G542X/1618T & & & & & & $1.14 \%$ & & & \\
\hline F508del/- & & & & & & $31.82 \%$ & & & \\
\hline G542X/- & & & & & & $1.14 \%$ & & & \\
\hline $\mathrm{R} 1162 \mathrm{X} /-$ & & & & & & $1.14 \%$ & & & \\
\hline$-/-$ & & & & & & $1.14 \%$ & & & \\
\hline
\end{tabular}

IFRD1 = Interferon-Related Developmental Regulator $1 ; C F T R=$ Cystic fibrosis transmembrane conductance regulator; $\mathrm{C}=\mathrm{Cytosine} ; \mathrm{T}=\mathrm{Thymine} ; \mathrm{z}=$ bigger than; $\mathrm{MAF}=$ minor allele frequency; ${ }^{*} \mathrm{p}=$ value for Hardy-Weinberg Equilibrium; $\mathrm{N}=$ number of patients; (-) CFTR mutation no identified. $1=I F R D 1, \mathrm{rs} 7817$ polymorphism is in Hardy-Weinberg Equilibrium in our sample.

Table 2: Genotypic characteristic of IFRD1 polymorphism and CFTR mutation among Cystic Fibrosis patients. 
Citation: de Lima Marson FA, Bariani Marcelino AR, Rezende LM, Ribeiro AF, Ribeiro JD, et al. (2013) The IFRD1 (57460C>T Polymorphism) Gene: A Negative Report in Cystic Fibrosis Clinical Severity. J Mol Genet Med 7: 058. doi:10.4172/1747-0862.1000058

Page 4 of 8

\begin{tabular}{|c|c|c|c|c|}
\hline \multirow{2}{*}{ Variables } & \multicolumn{2}{|c|}{ Without taking CFTR mutation into account } & \multicolumn{2}{|c|}{ Patients with two CFTR mutations identified } \\
\hline & $\mathbf{p}$ & $\mathbf{p}^{\mathrm{c}}$ & $\mathbf{p}$ & $\mathbf{p}^{\mathrm{c}}$ \\
\hline Sex ${ }^{1}$ & 0.765 & 1 & 0.754 & 1 \\
\hline Race $^{1}$ & 0.882 & 1 & 0.236 & 0.472 \\
\hline Age $^{1}$ & 0.992 & 1 & 0.925 & 1 \\
\hline Onset of symptoms ${ }^{1}$ & 0.526 & 1 & 0.929 & 1 \\
\hline Onset of pulmonary disease ${ }^{1}$ & 0.666 & 1 & 0.607 & 1 \\
\hline Onset of digestive disease ${ }^{1}$ & 0.595 & 1 & 0.793 & 1 \\
\hline Diagnosis $^{1}$ & 0.242 & 0.484 & 0.277 & 0.554 \\
\hline $\mathrm{BMI}^{1}$ & 0.740 & 1 & 0.859 & 1 \\
\hline Bhalla score $^{2}$ & 0.170 & 0.340 & 0.333 & 0.666 \\
\hline Kanga score ${ }^{2}$ & 0.913 & 1 & 0.828 & 1 \\
\hline Shwachman-Kulczycki score ${ }^{2}$ & 0.899 & 1 & 0.446 & 0.892 \\
\hline Nasal polyposis ${ }^{1}$ & 0.910 & 1 & 0.854 & 1 \\
\hline Diabetes melittus $^{1}$ & 0.531 & 1 & 0.891 & 1 \\
\hline Osteoporosis $^{1}$ & 0.502 & 1 & 0.669 & 1 \\
\hline Meconium ileous $^{1}$ & 0.750 & 1 & 0.669 & 1 \\
\hline Insufficiency pancreatic ${ }^{1}$ & 0.331 & 0.662 & 0.436 & 0.872 \\
\hline $\mathrm{SpO}_{2}^{2}$ & 0.342 & 0.684 & 0.684 & 1 \\
\hline $\mathrm{FVC}(\%)^{2}$ & 0.036 & 0.072 & 0.100 & 0.200 \\
\hline $\mathrm{FEV}_{1}(\%)^{2}$ & 0.142 & 0.284 & 0.153 & 0.306 \\
\hline $\mathrm{FEV}_{1} / \mathrm{FVC}^{2}$ & 0.838 & 1 & 0.838 & 1 \\
\hline FEF $_{25-75} \%{ }^{2}$ & 0.517 & 1 & 0.459 & 0.918 \\
\hline 1st $P$. aeruginosa ${ }^{1}$ & 0.377 & 0.754 & 0.541 & 1 \\
\hline$P$. aeruginosa mucoid ${ }^{1}$ & 0.553 & 1 & 0.517 & 1 \\
\hline$P$. aeruginosa no mucoid ${ }^{1}$ & 0.230 & 0.460 & 0.797 & 1 \\
\hline A. xylosoxidans ${ }^{1}$ & 0.502 & 1 & 0.888 & 1 \\
\hline S. aureus ${ }^{1}$ & 0.383 & 0.766 & 0.647 & 1 \\
\hline B. cepacia ${ }^{1}$ & 0.344 & 0.688 & 0.269 & 0.538 \\
\hline
\end{tabular}

IFRD1 = Interferon-Related Developmental Regulator 1; CFTR = Cystic Fibrosis Transmembrane Conductance Regulator; BMI = Body Mass Index; SpO2 = transcutaneous hemoglobin oxygen saturation; FVC - Forced Vital Capacity; FEV - Forced Expiratory Volume in the first second; FEF

FVC. $p=p$-value to statistical tests. $p^{c}=p$-value to statistical tests corrected by Bonferroni test. 'The positive $p$-value is in bold. 1 . Categorical variables $-X^{2}$ test was used. 2. Numerical variables - One-way analysis of variance test was used.

Table 3: Clinical association of cystic fibrosis variables with IFRD1 polymorphism (rs7817) and CFTR mutation

\begin{tabular}{|c|c|c|c|c|c|c|c|c|}
\hline \multirow{3}{*}{$\begin{array}{l}\text { IFRD1 gene } \\
\text { Genotype }\end{array}$} & \multicolumn{4}{|c|}{ Without taking CFTR mutation into account } & \multicolumn{4}{|c|}{ Two CFTR mutation identified } \\
\hline & \multicolumn{2}{|c|}{ Sex } & \multirow{2}{*}{ Total } & \multirow{2}{*}{$\mathrm{p}^{\mathrm{c}}$} & \multicolumn{2}{|c|}{ Sex } & \multirow{2}{*}{ Total } & \multirow{2}{*}{$\mathrm{p}^{c}$} \\
\hline & Female & Male & & & Female & Male & & \\
\hline $\mathrm{CC}$ & 14 & 14 & 28 & \multirow{3}{*}{1} & 9 & 10 & 19 & \multirow{3}{*}{1} \\
\hline CT & 19 & 16 & 35 & & 11 & 8 & 19 & \\
\hline TT & 15 & 10 & 25 & & 11 & 8 & 19 & \\
\hline \multirow{2}{*}{ Genotype } & \multicolumn{2}{|c|}{ Race } & \multirow{2}{*}{ Total } & \multirow{2}{*}{$p^{c}$} & \multicolumn{2}{|c|}{ Race } & \multirow{2}{*}{ Total } & \multirow{2}{*}{$p^{c}$} \\
\hline & Caucacasian & No caucasian & & & Caucacasian & No caucasian & & \\
\hline $\mathrm{CC}$ & 26 & 2 & 28 & \multirow{3}{*}{1} & 17 & 2 & 19 & \multirow{3}{*}{0.472} \\
\hline CT & 33 & 2 & 35 & & 18 & 1 & 19 & \\
\hline TT & 24 & 1 & 25 & & 19 & 0 & 19 & \\
\hline \multirow{2}{*}{ Genotype } & \multicolumn{2}{|c|}{ Age } & \multirow{2}{*}{ Total } & \multirow{2}{*}{$\mathrm{p}^{\mathrm{c}}$} & \multicolumn{2}{|c|}{ Age } & Total & \multirow{2}{*}{$p^{c}$} \\
\hline & $\leq 154$ months & $>154$ months & & & $\leq 154$ months & $>154$ months & & \\
\hline CC & 18 & 10 & 28 & \multirow{3}{*}{1} & 13 & 6 & 19 & \multirow{3}{*}{1} \\
\hline CT & 22 & 13 & 35 & & 13 & 6 & 19 & \\
\hline TT & 16 & 9 & 25 & & 12 & 7 & 19 & \\
\hline \multirow{2}{*}{ Genotype } & \multicolumn{2}{|c|}{ First clinical manifestation } & \multirow{2}{*}{ Total } & \multirow{2}{*}{$p^{c}$} & \multicolumn{2}{|c|}{ First clinical manifestation } & Total & \multirow{2}{*}{$\mathrm{p}^{\mathrm{c}}$} \\
\hline & $\leq 3$ months & $>3$ months & & & $\leq 3$ months & $>3$ months & Iotal & \\
\hline $\mathrm{CC}$ & 19 & 9 & 28 & & 12 & 7 & 19 & \\
\hline $\mathrm{CT}$ & 19 & 15 & 34 & 1 & 11 & 8 & 19 & 1 \\
\hline TT & 17 & 8 & 25 & & 11 & 8 & 19 & \\
\hline
\end{tabular}


Citation: de Lima Marson FA, Bariani Marcelino AR, Rezende LM, Ribeiro AF, Ribeiro JD, et al. (2013) The IFRD1 (57460C>T Polymorphism) Gene: A Negative Report in Cystic Fibrosis Clinical Severity. J Mol Genet Med 7: 058. doi:10.4172/1747-0862.1000058

Page 5 of 8

\begin{tabular}{|c|c|c|c|c|c|c|c|c|}
\hline \multirow{2}{*}{ Genotype } & \multicolumn{2}{|c|}{ Diagnosis } & \multirow{2}{*}{ Total } & \multirow{2}{*}{$\mathbf{p}^{c}$} & \multicolumn{2}{|c|}{ Diagnosis } & \multirow{2}{*}{ Total } & \multirow{2}{*}{$\mathbf{p}^{\mathrm{c}}$} \\
\hline & $\leq 24$ months & $>24$ months & & & $\leq 24$ months & $>24$ months & & \\
\hline $\mathrm{CC}$ & 16 & 10 & 26 & \multirow{3}{*}{0.484} & 10 & 8 & 18 & \multirow{3}{*}{0.554} \\
\hline CT & 21 & 13 & 34 & & 14 & 5 & 19 & \\
\hline TT & 20 & 5 & 25 & & 15 & 4 & 19 & \\
\hline \multirow{2}{*}{ Genotype } & \multicolumn{2}{|c|}{ First digestive manifestation } & \multirow{2}{*}{ Total } & \multirow{2}{*}{$\mathrm{p}^{\mathrm{c}}$} & \multicolumn{2}{|c|}{ First digestive manifestation } & \multirow{2}{*}{ Total } & \multirow{2}{*}{$\mathrm{p}^{\mathrm{c}}$} \\
\hline & $\leq 3$ months & $>3$ months & & & $\leq 3$ months & $>3$ months & & \\
\hline $\mathrm{CC}$ & 16 & 10 & 26 & \multirow{3}{*}{1} & 10 & 8 & 18 & \multirow{3}{*}{1} \\
\hline CT & 16 & 17 & 33 & & 11 & 8 & 19 & \\
\hline TT & 13 & 12 & 25 & & 9 & 10 & 19 & \\
\hline \multirow{2}{*}{ Genotype } & \multicolumn{2}{|c|}{ First pulmonary manifestation } & \multirow{2}{*}{ Total } & \multirow{2}{*}{$\mathrm{p}^{\mathrm{c}}$} & \multicolumn{2}{|c|}{ First pulmonary manifestation } & \multirow{2}{*}{ Total } & \multirow{2}{*}{$\mathrm{p}^{\mathrm{c}}$} \\
\hline & $\leq 6$ months & $>6$ months & & & $\leq 6$ months & $>6$ months & & \\
\hline $\mathrm{CC}$ & 18 & 10 & 28 & \multirow{3}{*}{1} & 12 & 7 & 19 & \multirow{3}{*}{1} \\
\hline CT & 19 & 15 & 34 & & 9 & 10 & 19 & \\
\hline TT & 16 & 8 & 24 & & 11 & 8 & 19 & \\
\hline \multirow{2}{*}{ Genotype } & \multicolumn{2}{|c|}{ Body mass index } & \multirow{2}{*}{ Total } & \multirow{2}{*}{$\mathrm{p}^{\mathrm{c}}$} & \multicolumn{2}{|c|}{ Body mass index } & \multirow{2}{*}{ Total } & \multirow{2}{*}{$\mathrm{p}^{\mathrm{c}}$} \\
\hline & 0 & 1 & & & 0 & 1 & & \\
\hline $\mathrm{CC}$ & 5 & 23 & 28 & \multirow{3}{*}{1} & 3 & 16 & 19 & \\
\hline СТ & 4 & 31 & 35 & & 2 & 17 & 19 & 0.1 \\
\hline TT & 3 & 22 & 25 & & 3 & 16 & 19 & \\
\hline
\end{tabular}

IFRD1 = Interferon-Related Developmental Regulator $1 ;$ CFTR $=$ Cystic Fibrosis Transmembrane Conductance Regulator; $\mathrm{p}^{\mathrm{c}}=\mathrm{p}$-value to statistical tests corrected by Bonferroni test; $\leq=$ minor than; $>=$ bigger than; $C=$ Cytosine; $T=$ Thymine; $0=$ thinness and accentuated thinness; $1=$ overweight/obesity and eutrophy.

Table 4: Association between IFRD1 polymorphism with clinical variables: sex, race, age, first clinical manifestation, time for diagnosis, time for the first digestive and pulmonary clinical manifestation and body mass index.

\begin{tabular}{|c|c|c|c|c|c|c|c|c|}
\hline \multirow{3}{*}{\begin{tabular}{|l} 
IFRD1 gene \\
Genotype
\end{tabular}} & \multicolumn{4}{|c|}{ Without taking CFTR mutation into account } & \multicolumn{4}{|c|}{ Two CFTR mutation identified } \\
\hline & \multicolumn{2}{|c|}{ Nasal polyposis } & \multirow{2}{*}{ Total } & \multirow{2}{*}{$\mathrm{p}^{c}$} & \multicolumn{2}{|c|}{ Nasal polyposis } & \multirow{2}{*}{ Total } & \multirow{2}{*}{$\mathrm{p}^{\mathrm{c}}$} \\
\hline & Absence & Presence & & & Absence & Presence & & \\
\hline CC & 25 & 3 & 28 & \multirow{3}{*}{1} & 17 & 2 & 19 & \multirow{3}{*}{1} \\
\hline CT & 30 & 5 & 35 & & 16 & 3 & 19 & \\
\hline TT & 22 & 3 & 25 & & 17 & 2 & 19 & \\
\hline \multirow{2}{*}{ Genotype } & \multicolumn{2}{|c|}{ Diabetes mellitus } & Tat & \multirow{2}{*}{$\mathrm{p}^{\mathrm{c}}$} & \multicolumn{2}{|c|}{ Diabetes mellitus } & \multirow{2}{*}{ Total } & \multirow{2}{*}{$p^{c}$} \\
\hline & Absence & Presence & Iotal & & Absence & Presence & & \\
\hline $\mathrm{CC}$ & 21 & 7 & 28 & \multirow{3}{*}{1} & 15 & 4 & 19 & \multirow{3}{*}{1} \\
\hline CT & 30 & 5 & 35 & & 16 & 3 & 19 & \\
\hline TT & 21 & 4 & 25 & & 15 & 4 & 19 & \\
\hline \multirow{2}{*}{ Genotype } & \multicolumn{2}{|c|}{ Osteoporosis } & Tatol & \multirow{2}{*}{$\mathrm{p}^{\mathrm{c}}$} & \multicolumn{2}{|c|}{ Osteoporosis } & \multirow{2}{*}{ Total } & \multirow{2}{*}{$p^{c}$} \\
\hline & Absence & Presence & I otal & & Absence & Presence & & \\
\hline $\mathrm{CC}$ & 22 & 6 & 28 & \multirow{3}{*}{1} & 15 & 4 & 19 & \multirow{3}{*}{1} \\
\hline СT & 31 & 4 & 35 & & 17 & 2 & 19 & \\
\hline TT & 22 & 3 & 25 & & 16 & 3 & 19 & \\
\hline \multirow{2}{*}{ Genotype } & \multicolumn{2}{|c|}{ Pancreatic insufficiency } & & \multirow{2}{*}{$\mathrm{p}^{\mathrm{c}}$} & \multicolumn{2}{|c|}{ Pancreatic insufficiency } & \multirow{2}{*}{ Total } & $n_{0}$ \\
\hline & Absence & Presence & Total & & Absence & Presence & & $\mathrm{p}^{\mathrm{c}}$ \\
\hline $\mathrm{CC}$ & 1 & 27 & 28 & & 1 & 18 & 19 & \\
\hline CT & 2 & 33 & 35 & 0.662 & 1 & 18 & 19 & 0.872 \\
\hline TT & 0 & 25 & 25 & & 0 & 19 & 19 & \\
\hline & Mec & ileus & & & Mec & ileus & Total & \\
\hline Genotype & Absence & Presence & Total & $\mathrm{p}^{\mathrm{c}}$ & Absence & Presence & Total & $p^{c}$ \\
\hline $\mathrm{CC}$ & 22 & 6 & 28 & & 15 & 4 & 19 & \\
\hline CT & 30 & 5 & 35 & 1 & 16 & 3 & 19 & 1 \\
\hline TT & 21 & 4 & 25 & & 17 & 2 & 19 & \\
\hline
\end{tabular}

IFRD1 = Interferon-Related Developmental Regulator $1 ;$ CFTR $=$ Cystic Fibrosis Transmembrane Conductance Regulator; $\mathrm{p}^{\mathrm{c}}=\mathrm{p}$-value to statistical tests corrected by Bonferroni test; $\mathrm{C}=$ Cytosine; $\mathrm{T}=$ Thymine.

Table 5: Association between IFRD1 polymorphism with comorbidities: nasal polyposis, diabetes mellitus, osteoporosis, pancreatic insufficiency and meconium ileus. 


\begin{tabular}{|c|c|c|c|c|c|c|c|c|}
\hline \multirow{3}{*}{\begin{tabular}{|l|} 
IFRD1 gene \\
Genotype \\
\end{tabular}} & \multicolumn{4}{|c|}{ Without taking CFTR mutation into account } & \multicolumn{4}{|c|}{ Two CFTR mutation identified } \\
\hline & \multicolumn{2}{|c|}{ First $P$. aeruginosa } & \multirow{2}{*}{ Total } & \multirow{2}{*}{$\mathrm{p}^{\mathrm{c}}$} & \multicolumn{2}{|c|}{ First $P$. aeruginosa } & \multirow{2}{*}{ Total } & \multirow{2}{*}{$\mathrm{p}^{\mathrm{c}}$} \\
\hline & $\leq 31$ months & $>31$ months & & & $\leq 31$ months & $>31$ months & & \\
\hline $\mathrm{CC}$ & 14 & 10 & 24 & \multirow{3}{*}{0.754} & 10 & 6 & 16 & \multirow{3}{*}{1} \\
\hline CT & 14 & 14 & 28 & & 7 & 8 & 15 & \\
\hline TT & 14 & 6 & 20 & & 11 & 6 & 17 & \\
\hline \multirow{2}{*}{ Genotype } & \multicolumn{2}{|c|}{ MPA } & Total & \multirow{2}{*}{$\mathrm{p}^{\mathrm{c}}$} & \multicolumn{2}{|c|}{ MPA } & \multirow{2}{*}{ Total } & \multirow{2}{*}{$p^{c}$} \\
\hline & Absence & Presence & Iotal & & Absence & Presence & & \\
\hline $\mathrm{CC}$ & 13 & 15 & 28 & \multirow{3}{*}{1} & 10 & 9 & 19 & \multirow{3}{*}{1} \\
\hline CT & 21 & 14 & 35 & & 13 & 6 & 19 & \\
\hline TT & 13 & 12 & 25 & & 10 & 9 & 19 & \\
\hline \multirow{2}{*}{ Genotype } & \multicolumn{2}{|c|}{ NMPA } & \multirow{2}{*}{ Total } & & \multicolumn{2}{|c|}{ NMPA } & \multirow{2}{*}{ Total } & \multirow{2}{*}{$p^{c}$} \\
\hline & Absence & Presence & & & Absence & Presence & & \\
\hline $\mathrm{CC}$ & 7 & 21 & 28 & \multirow{3}{*}{0.460} & 6 & 13 & 19 & \multirow{3}{*}{1} \\
\hline CT & 16 & 19 & 35 & & 8 & 11 & 19 & \\
\hline TT & 9 & 16 & 25 & & 7 & 12 & 19 & \\
\hline \multirow{2}{*}{ Genotype } & \multicolumn{2}{|c|}{ Achromobacter xylosoxidans } & Total & & Achromo & kylosoxidans & Total & $n^{c}$ \\
\hline & Absence & Presence & Iotal & & Absence & Presence & Iotal & $p^{c}$ \\
\hline $\mathrm{CC}$ & 22 & 6 & 28 & & 16 & 3 & 19 & \\
\hline CT & 31 & 4 & 35 & 1 & 15 & 4 & 19 & 1 \\
\hline TT & 22 & 3 & 25 & & 16 & 3 & 19 & \\
\hline Cenotvne & Burkh & pacia & & $n^{c}$ & Burkl & cepacia & Total & \\
\hline Genotype & Absence & Presence & Total & $\mathrm{p}^{\mathrm{c}}$ & Absence & Presence & Total & $p^{c}$ \\
\hline $\mathrm{CC}$ & 25 & 3 & 28 & & 17 & 2 & 19 & \\
\hline CT & 27 & 8 & 35 & 0.688 & 13 & 6 & 19 & 0.538 \\
\hline TT & 19 & 6 & 25 & & 15 & 4 & 19 & \\
\hline & Staphyl & aureus & & & Staph & us aureus & & \\
\hline Genotype & Absence & Presence & Iotal & $\mathrm{p}^{\mathrm{c}}$ & Absence & Presence & Iotal & $p^{c}$ \\
\hline $\mathrm{CC}$ & 6 & 22 & 28 & & 5 & 14 & 19 & \\
\hline Ст & 4 & 31 & 35 & 0.766 & 3 & 16 & 19 & 1 \\
\hline TT & 6 & 19 & 25 & & 3 & 16 & 19 & \\
\hline
\end{tabular}

IFRD1 $=$ Interferon-Related Developmental Regulator 1; CFTR $=$ Cystic Fibrosis Transmembrane Conductance Regulator; $\mathrm{p}^{\mathrm{c}}=\mathrm{p}$-value to statistical tests corrected by Bonferroni test; $\mathrm{C}=$ Cytosine; $\mathrm{T}=$ Thymine; $\leq=$ minor than; $>=$ bigger than; MPA = mucoid $P$. aeruginosa; NMPA = non-mucoid $P$. aeruginosa; $\mathrm{AX}=$ Achromobacter xylosoxidans; $\mathrm{BC}=$ Burkholderia cepacia; $\mathrm{SA}=$ Staphylococcus aureus.

Table 6: Association between IFRD1 polymorphism with bacteria on sputum.

two mutations identified belonging to class I, II and III. Categorical variables are described in absolute frequency and numerical by mean, standard deviation, minimum and maximum value, and confidence interval. In the tables 4-7, p-corrected values are presented.

\section{Discussion}

The evolution of $\mathrm{CF}$ as a disease is the result of the interaction between genotype and environment. Few studies have correlated CFTR mutations, modifier genes and clinical variables in CF [2,6,7,26], a fact associated with the difficulty in obtaining: (i) sample size, (ii) patients with homogeneous treatment, and (iii) to characterize the follow-up of pulmonary disease.

The principal environmental factor for the clinical variability of CF is the treatment access. In our referral center, treatment is warranted for the public health system, which allows equal access for all patients included in the study, and no concerns as an additional factor in the statistical analyzes.

The IFRD1 protein expression is not restricted to neutrophils, but may also occur in epithelial cells in organs that compose the airways acting in the inflammatory process, having an important role in the pulmonary disease [27]. However, few studies have compared the expression and regulation of IFRD1 in different cellular types in order to understand the complex development of lung disease, hence, more studies are needed $[17,18,27,28]$.

Two polymorphisms (rs11771128 and rs4727770) in the IFRD1 gene were associated with CF modulation [28]. Heterozygous patients for the polymorphism had higher levels of IFRD1 in neutrophils from the bloodstream, compared to homozygotes. However, it is still unclear how the differential expression influences and governs the defense system is still unclear.

The neutrophil regulation is important in the inflammatory process, which is the basis of the pathophysiology of the CF pulmonary manifestations, thus the role of the IFRD1 protein can have influence on the CF severity [17]. In this sense, the analysis by array for $320 \mathrm{CF}$ patients divided into two groups according to clinical severity showed that IFRD1 polymorphisms could function as modulators of clinical severity [20].

Other studies [17,18] have found a relationship of $57460 \mathrm{C}$ polymorphism in IFRD1 gene and the severity of lung disease in children and adolescents CF patients.

In our study, we did not find this association, even considering the 
Citation: de Lima Marson FA, Bariani Marcelino AR, Rezende LM, Ribeiro AF, Ribeiro JD, et al. (2013) The IFRD1 (57460C>T Polymorphism) Gene: A Negative Report in Cystic Fibrosis Clinical Severity. J Mol Genet Med 7: 058. doi:10.4172/1747-0862.1000058

Page 7 of 8

\begin{tabular}{|c|c|c|c|c|c|c|c|c|c|}
\hline \multirow{2}{*}{ Variable } & \multirow{2}{*}{$\begin{array}{c}\text { IFRD1 } \\
\text { genotype }\end{array}$} & \multirow{2}{*}{$\mathbf{N}$} & \multirow{2}{*}{ Mean } & \multirow{2}{*}{ Std. Deviation } & \multicolumn{2}{|c|}{ 95\% Confidential Interval } & \multirow{2}{*}{ Min } & \multirow{2}{*}{$\operatorname{Max}$} & \multirow{2}{*}{$\mathbf{p}^{\mathrm{c}}$} \\
\hline & & & & & Lower Bound & Upper Bound & & & \\
\hline \multirow{3}{*}{$\mathrm{SpO} 2$} & $\mathrm{CC}$ & 28 & 94.54 & 6.173 & 92.14 & 96.93 & 66 & 98 & \multirow{3}{*}{0.684} \\
\hline & CT & 34 & 95.79 & 2.544 & 94.91 & 96.68 & 87 & 99 & \\
\hline & TT & 25 & 96.08 & 2.871 & 94.89 & 97.27 & 86 & 99 & \\
\hline \multirow{3}{*}{ Bhalla } & $\mathrm{CC}$ & 21 & 9.76 & 3.590 & 8.13 & 11.40 & 6 & 23 & \multirow{3}{*}{0.340} \\
\hline & CT & 22 & 7.23 & 4.503 & 5.23 & 9.22 & 0 & 23 & \\
\hline & TT & 19 & 7.74 & 5.496 & 5.09 & 10.39 & 0 & 22 & \\
\hline \multirow{3}{*}{ Kanga } & $\mathrm{CC}$ & 26 & 18.46 & 5.770 & 16.13 & 20.79 & 10 & 36 & \multirow{3}{*}{1} \\
\hline & CT & 30 & 17.97 & 5.512 & 15.91 & 20.02 & 11 & 33 & \\
\hline & TT & 22 & 18.64 & 6.730 & 15.65 & 21.62 & 12 & 40 & \\
\hline \multirow{3}{*}{$\begin{array}{l}\text { Shwachman- } \\
\text { Kulczycki }\end{array}$} & $\mathrm{CC}$ & 26 & 66.92 & 12.496 & 61.88 & 71.97 & 40 & 85 & \multirow{3}{*}{1} \\
\hline & CT & 28 & 68.75 & 13.026 & 63.70 & 73.80 & 40 & 90 & \\
\hline & TT & 24 & 67.92 & 17.871 & 60.37 & 75.46 & 20 & 90 & \\
\hline \multirow{3}{*}{ FVC\% } & $\mathrm{CC}$ & 22 & 72.09 & 17.318 & 64.41 & 79.77 & 29 & 105 & \multirow{3}{*}{0.072} \\
\hline & CT & 22 & 87.18 & 18.887 & 78.81 & 95.56 & 58 & 131 & \\
\hline & TT & 16 & 84.38 & 24.055 & 71.56 & 97.19 & 41 & 135 & \\
\hline & $\mathrm{CC}$ & 22 & 65.36 & 20.127 & 56.44 & 74.29 & 19 & 114 & \\
\hline $\mathrm{FEV}_{1} \%$ & CT & 21 & 78.67 & 24.836 & 67.36 & 89.97 & 36 & 132 & 0.284 \\
\hline & TT & 16 & 75.19 & 22.013 & 63.46 & 86.92 & 27 & 100 & \\
\hline & $\mathrm{CC}$ & 22 & 81.95 & 16.114 & 74.81 & 89.10 & 37 & 102 & \\
\hline $\mathrm{FEV}_{1} / \mathrm{FVC}$ & CT & 21 & 83.90 & 11.291 & 78.77 & 89.04 & 58 & 99 & 1 \\
\hline & TT & 16 & 84.31 & 11.780 & 78.04 & 90.59 & 59 & 99 & \\
\hline & $\mathrm{CC}$ & 22 & 53.09 & 31.355 & 39.19 & 66.99 & 8 & 134 & \\
\hline FEF $_{25-75} \%$ & $\mathrm{CT}$ & 21 & 63.57 & 33.817 & 48.18 & 78.96 & 13 & 121 & 1 \\
\hline & TT & 16 & 60.44 & 23.639 & 47.84 & 73.03 & 11 & 88 & \\
\hline & $\mathrm{CC}$ & 19 & 94.63 & 7.259 & 91.13 & 98.13 & 66 & 98 & \\
\hline $\mathrm{SpO} 2$ & CT & 18 & 95.78 & 2.045 & 94.76 & 96.79 & 92 & 98 & 1 \\
\hline & TT & 19 & 95.84 & 3.253 & 94.27 & 97.41 & 86 & 99 & \\
\hline & $\mathrm{CC}$ & 15 & 9.40 & 3.961 & 7.210 & 11.59 & 6 & 23 & \\
\hline Bhalla & CT & 11 & 6.73 & 3.319 & 4.500 & 8.96 & 0 & 10 & 0.666 \\
\hline & TT & 16 & 7.94 & 5.603 & 4.950 & 10.92 & 0 & 22 & \\
\hline & $\mathrm{CC}$ & 18 & 18.06 & 6.197 & 14.97 & 21.14 & 10 & 36 & \\
\hline Kanga & CT & 15 & 18.53 & 6.243 & 15.08 & 21.99 & 11 & 33 & 1 \\
\hline & TT & 16 & 19.44 & 7.339 & 15.53 & 23.35 & 12 & 40 & \\
\hline & $\mathrm{CC}$ & 18 & 70.00 & 12.005 & 64.03 & 75.97 & 40 & 85 & \\
\hline $\begin{array}{l}\text { Shwachman- } \\
\text { Kulczycki }\end{array}$ & CT & 15 & 66.00 & 12.845 & 58.89 & 73.11 & 45 & 90 & 0.892 \\
\hline & TT & 18 & 63.89 & 17.703 & 55.09 & 72.69 & 20 & 90 & \\
\hline & $\mathrm{CC}$ & 16 & 73.31 & 18.930 & 63.23 & 83.40 & 29 & 105 & \\
\hline FVC & CT & 13 & 91.23 & 22.391 & 77.70 & 104.76 & 58 & 131 & 0.200 \\
\hline & TT & 14 & 83.93 & 24.765 & 69.63 & 98.23 & 41 & 135 & \\
\hline & $\mathrm{CC}$ & 16 & 66.00 & 21.404 & 54.59 & 77.41 & 19 & 114 & \\
\hline $\mathrm{FEV}_{1}$ & $\mathrm{CT}$ & 13 & 83.92 & 28.558 & 66.67 & 101.18 & 36 & 132 & 0.306 \\
\hline & TT & 14 & 74.57 & 22.779 & 61.42 & 87.72 & 27 & 100 & \\
\hline & $\mathrm{CC}$ & 16 & 83.00 & 17.278 & 73.79 & 92.21 & 37 & 102 & \\
\hline $\mathrm{FEV}_{1} / \mathrm{FVC}$ & $\mathrm{CT}$ & 13 & 86.15 & 12.694 & 78.48 & 93.82 & 58 & 99 & 1 \\
\hline & TT & 14 & 83.86 & 12.322 & 76.74 & 90.97 & 59 & 99 & \\
\hline & $\mathrm{CC}$ & 16 & 55.81 & 33.293 & 38.07 & 73.55 & 8 & 134 & \\
\hline FEF $_{25-75} \%$ & CT & 13 & 70.92 & 37.604 & 48.20 & 93.65 & 13 & 121 & 1 \\
\hline & TT & 14 & 60.86 & 25.301 & 46.25 & 75.47 & 11 & 88 & \\
\hline
\end{tabular}

IFRD1 = Interferon-Related Developmental Regulator 1; CFTR $=$ Cystic Fibrosis Transmembrane Conductance Regulator; $\mathrm{p}^{\mathrm{c}}=\mathrm{p}$-value to statistical tests corrected by Bonferroni test; $\mathrm{C}=$ Cytosine; $\mathrm{T}=$ Thymine; $\mathrm{N}=$ number of patients; $\min =$ minimum; $\max =$ maximum; std $=$ standard; $\mathrm{SpO}_{2}=\mathrm{Transcutaneous}$ oxygen saturation; $\mathrm{FVC}=$ forced vital capacity; $\mathrm{FEV}_{1}=$ forced expiratory volume in the first second; $\mathrm{FEF}_{25-75}=$ forced expiratory flow between 25 and $75 \%$ of $\mathrm{FVC}$.

Table 7: Association between IFRD1 polymorphism with clinical variables with numerical distribution: lung function and clinical scores. 
Citation: de Lima Marson FA, Bariani Marcelino AR, Rezende LM, Ribeiro AF, Ribeiro JD, et al. (2013) The IFRD1 (57460C>T Polymorphism) Gene: A Negative Report in Cystic Fibrosis Clinical Severity. J Mol Genet Med 7: 058. doi:10.4172/1747-0862.1000058

Page 8 of 8

23 variables of clinical severity. We expected that CF patients would show lower expression of the IFRD1 protein and that the results would have association with clinical variables, especially those associated with pulmonary disease. Our results differ from those of previous studies possibly because earlier studies (i) considered homogeneous populations, (ii) used fewer clinical markers, (iii) did not consider IFRD1 polymorphisms, but rather only the amount of IFRD1 protein, (iv) evaluated fewer patients.

\section{Conclusions}

We found that in our sample of CF patients, there was no association of the polymorphism $57460 \mathrm{C}$ in the IFRD1 gene with the disease severity. Studies considering the analysis of other polymorphisms within the same gene or other genes, as modifier gene, must be considered. However, it is still necessary to study polymorphisms achieve a better understanding of the dynamics of the clinical manifestations and clinical variability of the disease, even in individuals with the identical CFTR genotype.

\section{Competing Interests}

The authors declare that they have no competing interests.

\section{Acknowledgments}

Luciana Cardoso Bonadia, Taís Daiene Russo Hortêncio, Kátia Cristina Alberto Aguiar, Aline Gonçalves, Carlos Emilio Levy, Maria de Fátima Servidon and Simoni Avansini - assistance in data collection and organization of ideas. Maria Angela Ribeiro and Staff of LAFIP - (Laboratorio de Fisiologia Pulmonar) for spirometry analysis. To Fapesp: provide assistance to search. To www. laboratoriomultiussuario.com.br: to possibility the genetic analysis.

\section{References}

1. Knowles MR (2006) Gene modifiers of lung disease. Curr Opinin Pulm Med 12: $416-421$

2. Drumm ML, Ziady AG, Davis PB (2007) Genetic variation and clinical heterogeneity in cystic fibrosis. Annu Rev Pathol 7: 267-282.

3. Accurso FJ, Sontag MK (2008) Gene modifiers in cystic fibrosis. J Clin Invest 118: 839-841.

4. Blackman SM, Deering-Brose R, McWilliams R, Naughton $\mathrm{K}$, Coleman B, et al. (2006) Relative contribution of genetic and nongenetic modifiers to intestinal obstruction in cystic fibrosis. Gastroenterology 131: 1030-1039.

5. Boyle MP (2007) Strategies for identifying modifier genes in cystic fibrosis. Proc Am Thorac Soc 4: 52-57.

6. Knowles MR, Drumm M (2012) The influence of genetics on cystic fibrosis phenotypes. Cold Spring Harb Perspect Med 2: a009548.

7. Dorfman R (2012) Modifier gene studies to identify new therapeutic targets in cystic fibrosis. Curr Pharm Des 18: 674-682.

8. Grasemann H, Buscher R (2006) Disease modifying genes in cystic fibrosis therapeutic option or one-way road? Naunyn Schmiedebergs Arch Pharmaco 374: 65-77.

9. Faria EJ, Faria IC, Ribeiro JD, Ribeiro AF, Hessel G, et al. (2009) Association of MBL2, TGF-beta1 and CD14 gene polymorphisms with lung disease severity in cystic fibrosis. J Bras Pneumol 35: 334-342.

10. Lima CS, Ortega MM, Marson FA, Zulli R, Ribeiro AF, et al. (2012) Cystic fibrosis transmembrane conductance regulator gene mutations and glutathione S-transferase null genotypes in cystic fibrosis patients in Brazil. J Bras Pneumol 38: 50-56.

11. Marson FA, Bertuzzo CS, Hortencio TD, Ribeiro JD, Bonadia LC, et al. (2012) The ACE gene $\mathrm{D} / \mathrm{l}$ polymorphism as a modulator of severity of cystic fibrosis.
BMC Pulm Med 12: 41

12. Marson FA, Bertuzzo CS, Ribeiro AF, Ribeiro JD (2012) Polymorphisms in $A D R B 2$ gene can modulate the response to bronchodilators and the severity of cystic fibrosis. BMC Pulm Med 12: 50 .

13. Furgeri DT, Marson FA, Ribeiro AF, Bertuzzo CS (2012) Association between the IVS4G>T mutation in the TCF7L2 gene and susceptibility to diabetes in cystic fibrosis patients. BMC Res Notes 5: 561.

14. Marson FAL, Marcelino ARB, Ribeiro AF (2013) COX-2 Gene Polymorphisms: Genetic Determinants of Cystic Fibrosis Comorbidities. Int J Genet 5: 132-138.

15. Marson FAL, Rezende LM, Furgeri DT, Ribeiro AF, Ribeiro JD, et al. (2013) $A D R A 2 A$ is a Cystic Fibrosis Modifier Gene. International Journal of Genetics 5: 125-131.

16. Buanne P, Incerti B, Guardavaccaro D, Avvantaggiato V, Simeone A, et al. (1998) Cloning of the human interferon-related developmental regulator (IFRD1) gene coding for the PC4 protein, a member of a novel family of developmentally regulated genes. Genomics 51: 233-242.

17. Gu Y, Harley IT, Henderson LB, Aronow BJ, Vietor I, et al. (2009) Identification of IFRD1 as a modifier gene for cystic fibrosis lung disease. Nature 458: 10391042

18. Ehrnhoefer DE (2009) IFRD1 modulates disease severity in cystic fibrosis through the regulation of neutrophil effector function. Clin Genet 76: 148-149.

19. Vadivelu SK, Kurzbauer R, Dieplinger B, Zweyer M, Schafer R, et al. (2004) Muscle regeneration and myogenic differentiation defects in mice lacking TIS7. Mol Cell Biol 24: 3514-3525.

20. Kerem E, Corey M, Kerem BS, Rommens J, Markiewicz D, et al. (1990) The relation between genotype and phenotype in cystic fibrosis: analysis of the most common mutation (delta F508). N Engl J Med 323: 1517-1522.

21. Santos CIS, Ribeiro JD, Ribeiro AF, Hessel G (2004) Critical analysis of scoring systems used in the assessment of Cystic Fibrosis severity: state of the art. J Bras Pneumol 30: 286-298.

22. American Thoracic Society.

23. Dean AG, Sullivan KM, Soe MM (2011) OpenEpi: Open Source Epidemiologic Statistics for Public Health, Version 2.3.1.

24. Faul F, Erdfelder E, Buchner A, Lang AG (2009) Statistical power analyses using $\mathrm{G}^{*}$ Power 3.1: Tests for correlation and regression analyses. Behav Res Methods 41: 1149-1160.

25. Drăghici S (2003) Data analysis tools for DNA microarrays. Chapman \& Hall/ CRC, New York

26. Cutting GR (2010) Modifier genes in Mendelian disorders: the example of cystic fibrosis. Ann N Y Acad Sci 1214: 57-69.

27. Blanchard E, Marie S, Riffault L, Bonora M, Tabary O, et al. (2011) Reduced expression of Tis7/IFRD1 protein in murine and human cystic fibrosis airway epithelial cell models homozygous for the F508del-CFTR mutation. Biochem Biophys Res Commun 411: 471-476.

28. Hector A, Kormann M, Kammermeier J, Burdi S, Marcos V, et al. (2013) Expression and regulation of interferon-related development regulator-1 in cystic fibrosis neutrophils. Am J Respir Cell Mol Biol 48: 71-77. 